\title{
The XMM-Newton and BeppoSAX view of the Ultra Luminous Infrared Galaxy MKN 231
}

\author{
V. Braito ${ }^{1,2}$, R. Della Ceca ${ }^{1}$, E. Piconcelli ${ }^{3,4}$, P. Severgnini ${ }^{1}$, L. Bassani ${ }^{3}$, M. Cappi ${ }^{3}$, A. Franceschini ${ }^{2}$, K. Iwasawa $^{5}$,

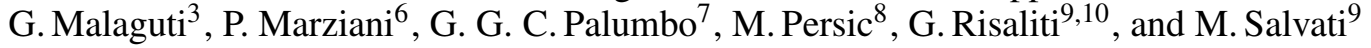 \\ 1 INAF - Osservatorio Astronomico di Brera, Milano, Italy \\ 2 Dipartimento di Astronomia, Università di Padova, Italy \\ ${ }^{3}$ IASF - CNR, Bologna, Italy \\ 4 XMM-SOC (VILSPA), ESA, Madrid, Spain \\ 5 Institute of Astronomy, University of Cambridge, Madingley Road Cambridge CB3 OHA, UK \\ 6 INAF - Osservatorio Astronomico di Padova, Italy \\ 7 Dipartimento di Astronomia, Università di Bologna, Italy \\ 8 INAF - Osservatorio Astronomico di Trieste, Italy \\ 9 INAF - Osservatorio Astrofisico di Arcetri, Firenze, Italy \\ ${ }^{10}$ Harvard-Smithsonian Center for Astrophysics, Cambridge, USA
}

Received 28 May 2003 / Accepted 15 January 2004

\begin{abstract}
We discuss XMM-Newton and BeppoSAX observations of MKN 231, the lowest-redshift Broad Absorption Line (BAL) QSO known so far and one of the best-studied Ultra Luminous Infrared Galaxies. By combining the XMM-Newton spectral resolution and the high-energy bandpass of BeppoSAX we have been able to study in more detail than previously possible its $0.2-50 \mathrm{keV}$ spectral properties. The BeppoSAX PDS data unveiled, for the first time, a highly absorbed $\left(N_{\mathrm{H}} \sim 2 \times 10^{24} \mathrm{~cm}^{-2}\right)$ power-law component. We find that: a) below $10 \mathrm{keV}$ we are seeing only reprocessed radiation through reflection and/or scattering; b) the intrinsic 2-10 keV luminosity of MKN 231 is $1_{-0.5}^{+1.0} \times 10^{44} \mathrm{erg} \mathrm{s}^{-1}$, i.e. more than an order of magnitude greater than previous measurements; c) the starburst activity significantly contributes to the soft $(E<2 \mathrm{keV}) \mathrm{X}$-ray emission.
\end{abstract}

Key words. X-rays: galaxies - galaxies: individual: Markarian 231

\section{Introduction}

In the local $(z<0.05)$ Universe Markarian $231(z=0.042$, $L_{\text {bol }}=6 \times 10^{12} L_{\odot}$ for $\left.H_{0}=50 \mathrm{~km} \mathrm{~s}^{-1} \mathrm{Mpc}^{-1}\right)$ is one of the most luminous and best studied Ultra Luminous Infrared Galaxies (i.e. sources with $L_{\mathrm{IR}}>10^{12} L_{\odot}$; hereafter ULIRGs). This object is optically classified as a Broad Absorption Line (BAL) QSO (Smith et al. 1995). This class of sources, which represent $\sim 10 \%$ of the optically selected QSOs, are known to be weak X-ray sources, their faintness being attributed to intrinsic absorption (Green et al. 2001; Gallagher et al. 2002a).

Although the nature of the primary energy source (AGN vs. starburst activity) for the high luminosity of ULIRGs is still a debated issue (see Ptak et al. 2003; Franceschini et al. 2003, and references therein), in the case of MKN 231 optical and near infrared observations seem to suggest that a significant contribution to the infrared luminosity $\left(L_{\mathrm{FIR}} \simeq 6 \times 10^{12} L_{\odot}\right)$ could be ascribed to AGN activity (Goldader et al. 1995;

Send offprint requests to: $\mathrm{V}$. Braito,

e-mail: braito@brera.mi.astro.it
Krabbe et al. 1997). On the other hand this galaxy is also undergoing an energetic starburst and, in particular, radio observations of the inner kiloparsec regions suggest that a nuclear ring of active star formation is proceeding at a rate of $\sim 220 M_{\odot} \mathrm{yr}^{-1}$ (Taylor et al. 1999) ${ }^{1}$. Indication of a strong starburst activity, which can account for up to $70 \%$ of the $L_{\mathrm{FIR}}$ of MKN 231 (corresponding to an $S F R \sim 470 \pm 50 M_{\odot} \mathrm{yr}^{-1}$ ), comes also from the modelling of the radio to optical spectral energy distribution (Farrah et al. 2003). This latter estimate is in good agreement with the AGN fraction derived by Lonsdale et al. (2003) using VLBI observations at $18 \mathrm{~cm}$. As discussed by several authors the combination of starburst and luminous AGN makes MKN 231 one of the best examples of the transition from starburst to AGN according to the scheme outlined in Sanders et al. (1996).

The strong circumnuclear starburst activity together with the presence of high intrinsic absorption, probably associated

\footnotetext{
${ }^{1}$ In this respect it is worth noting that the starforming rate in M 82, the prototype local starburst galaxy, is about one order of magnitude lower (Grimm et al. 2003).
} 
with the BAL outflows (Green et al. 2001), makes the X-ray continuum of this source particularly complex. Many different spectral components should be taken into account in the spectral modeling i.e. the thermal and the binary emission associated with the products of the starburst as well as the scattered/reflected and intrinsic component(s) associated with the AGN.

Previous X-ray observations by ROSAT, ASCA (Turner 1999; Iwasawa 1999; Maloney \& Reynolds 2000, hereafter MR00) and more recently Chandra (Gallagher et al. 2002b, hereafter G02; Ptak et al. 2003), revealed the presence of: a) extended soft X-ray emission of thermal origin, likely associated with the circumnuclear starburst; b) a hard and flat (photon in$\operatorname{dex} \Gamma \sim 0.7)$ non-thermal component having an observed X-ray luminosity $L_{(2-10)} \sim 10^{42} \mathrm{erg} \mathrm{s}^{-1}$ and c) a Fe $\mathrm{K}_{\alpha}$ emission line having an observed equivalent width of $\sim 300 \mathrm{eV}$ (the line was measured by ASCA but is not detected by Chandra).

To explain the low observed luminosity (which is at least two orders of magnitude lower than that expected for an AGN of similar bolometric luminosity), the flat photon index and the $E W$ of the $\mathrm{Fe} \mathrm{K} \alpha$ line, MR00 suggested that about 3/4 of the observed 2-10 keV spectrum of MKN 231 consists of a scattered power-law component plus a reflected component (no direct AGN component is seen below $\sim 10 \mathrm{keV}$ ). Both reflected and scattered components are absorbed by an intrinsic column density of $N_{\mathrm{H}} \sim 3 \times 10^{22} \mathrm{~cm}^{-2}$. On the contrary G02, using Chandra data (which in addition make it possible to disentangle the nuclear from the extended component), support a model in which a small, Compton-thick absorber blocks the direct X-rays. Only indirect scattered emission from multiple lines of sight can reach the observer in the Chandra energy range; in particular they proposed a triple scattering model composed of three separate absorbed power laws having a photon index fixed to 2.1 and absorbing column densities of $N_{\mathrm{H}} \simeq 1.2 \times 10^{21} \mathrm{~cm}^{-2}, N_{\mathrm{H}} \simeq 3.2 \times 10^{22} \mathrm{~cm}^{-2}$ and $N_{\mathrm{H}} \simeq 5.9 \times 10^{23} \mathrm{~cm}^{-2}$, respectively. According to the modeling of MR00 and G02, the X-ray emission below $10 \mathrm{keV}$ is only reprocessed radiation, thus they could evaluate the intrinsic luminosity of MKN 231 only through indirect arguments.

Here the results from XMM-Newton and BeppoSAX observations of this object are presented. The main result from these data is that a highly absorbed $\left(N_{\mathrm{H}} \sim 2 \times 10^{24} \mathrm{~cm}^{-2}\right)$ AGN component having an intrinsic 2-10 keV luminosity of $1_{-0.5}^{+1.0} \times 10^{44} \mathrm{erg} \mathrm{s}^{-1}$ has been detected. This is the first direct measurement of the intrinsic power of the AGN hosted by MKN 231. This paper is organized as follows: observation and data analysis are described in Sect. 2; timing and spectral analysis are reported in Sects. 3 and 4. Results and discussion are presented in Sect. 5. In this paper we assume $H_{0}=50 \mathrm{~km} \mathrm{~s}^{-1} \mathrm{Mpc}^{-1}$ and $q_{0}=0.5$.

\section{Observations and data reduction}

MKN 231 is part of an XMM-Newton survey of 10 nearby $(z<0.2)$ ULIRGs for which high-quality mid-IR and optical spectroscopic data are available. Results on this project are presented and discussed in Braito et al. (2002) and in Franceschini et al. (2003). MKN 231 also belongs to a small sample of ULIRGs for which we have obtained BeppoSAX measurements.

\subsection{XMM-Newton data}

MKN 231 was targeted for $20 \mathrm{ks}$ by XMM-Newton on July 6th, 2001. The EPIC cameras (European Photon Imaging Camera: Strüder et al. 2001 and Turner et al. 2001) were operating in full-frame mode with the thin filter applied. Event files produced from the standard pipeline processing have been analyzed and filtered (using version 5.4 of the Science Analysis Software, SAS, and the latest calibration files released by the EPIC team) from high background time intervals and only events corresponding to pattern 0-12 for MOS and pattern 0-4 for pn have been used (see the XMM-Newton Users' Handbook, Ehle et al. 2001). The net exposure times, after data cleaning, are $\sim 16.0 \mathrm{ks}, \sim 19.9 \mathrm{ks}$ and $\sim 19.8 \mathrm{ks}$ for $\mathrm{pn}$, MOS1 and MOS2 respectively.

In the low-energy domain $(E<2 \mathrm{keV})$, the X-ray emission of MKN 231 appears to be extended ${ }^{2}$, with a roughly circular symmetry. The presence of extended soft X-ray emission was first noted using ROSAT observations (Turner 1999), and it has been confirmed by the recent Chandra observation (G02). The Chandra data show that the galaxy emission (0.35-2 keV range) extends over a radius of $\sim 25$ arcsec. The hard (2-10 keV) X-ray brightness profile is comparable to the $\mathrm{XMM}-$ Newton PSF, whose HEW at $\sim 8 \mathrm{keV}$ is $\sim 14^{\prime \prime}$.

Since in this paper we want to address the X-ray spectral properties of the nuclear and circumnuclear X-ray emission, source counts have been extracted from a circular region of 20 arcsec radius ${ }^{3}$, positionally coincident with the core of the hard X-ray emission. The X-ray position is also coincident (within the XMM-Newton positional accuracy $\sim$ few arcsec) with the core of the optical and radio emission. The XMM-Newton PSF does not allow us to select a smaller region and thus, although the extraction radius has been chosen to minimize the effect of the extended soft emission, in the derived spectrum we expect imprinted signatures of the strong circumnuclear starburst activity of MKN 231.

The presence of other sources around MKN 231 prevented the selection of a nearby large enough area to accumulate background counts with good statistics; therefore background counts have been accumulated using the background event files distributed by the EPIC team. Response matrices (that include the correction for the effective area) have been generated using the SAS tasks arfgen and rmfgen. The net count rate in the $0.2-10 \mathrm{keV}$ energy range is $(11 \pm 1) \times 10^{-2} \mathrm{cts} / \mathrm{s}$, $(3.77 \pm 0.14) \times 10^{-2} \mathrm{cts} / \mathrm{s}$ and $(3.54 \pm 0.14) \times 10^{-2} \mathrm{cts} / \mathrm{s}$ for $\mathrm{pn}$, MOS1 and MOS2, respectively.

\footnotetext{
2 A detailed spectral and imaging analysis of the extended component will be reported elsewhere.

3 The X-ray source detected by G02 using Chandra data at $3^{\prime \prime}$ apart from the nucleus is thus included in the counts extraction region. However, as its flux is two orders of magnitude lower than that of the nuclear emission of MKN 231, it does not affect the results reported here.
} 


\subsection{BeppoSAX data}

MKN 231 was observed by BeppoSAX (Boella et al. 1997) from December 29th, 2001 to January 1st, 2002. In this paper we use data collected from the Medium Energy Concentrator Spectrometer (MECS) and from the Phoswich Detector System (PDS). We do not use data from the Low Energy Concentrator Spectrometer since they are of significantly lower quality when compared with the low energy XMM-Newton data.

Standard data reduction was performed using the software package SAXDAS (see http://www.sdc.asi.it/ software and the Cookbook; Fiore et al. 1999) for the MECS datasets and XAS (Chiappetti \& Dal Fiume 1997) for the PDS data. Spectral fits were performed using public response matrices as from the 1998 November issue. BeppoSAX pointed at MKN 231 for an effective exposure time of $144 \mathrm{ks}$ and $76 \mathrm{ks}$ for MECS and PDS, respectively. To maximize statistics and signal-to-noise ratio, the MECS source counts were extracted from a circular region of 2 arcmin radius. The background spectrum was taken from blank sky fields at the position of the source with the same extraction radius. As suggested in the BeppoSAX Cookbook we have considered only the MECS data in the 1.8-10 keV energy range. Spectral channels corresponding to energies in the range $15-60 \mathrm{keV}$ were used for the PDS. MECS source counts have been rebinned proportionally to the instrumental resolution and so as to have always more than 20 counts per channel; the PDS data instead have been rebinned to maximize the signal to noise ratio in each energy bin. The net count rates are $(5.68 \pm 0.23) \times 10^{-3} \mathrm{cts} / \mathrm{s}$ in the MECS and $(6.58 \pm 2.16) \times 10^{-2} \mathrm{cts} / \mathrm{s}$ in the PDS.

\section{X-ray variability}

The Chandra data of MKN 231 discussed by G02 indicate significant variability with a count rate decrease of $\sim 45 \%$ on a $20 \mathrm{ks}$ time-scale at energies above $2 \mathrm{keV}$. This variability coupled with the $188 \mathrm{eV}$ upper limit on the $E W$ of the Fe K $\alpha$ emission line was used by G02 to argue against the reflection model proposed by MR00.

Thus we first investigated the variability properties of MKN 231 using the BeppoSAX data. Although BeppoSAX has a significantly worse spatial resolution than Chandra, the long exposure time ( $144 \mathrm{ks}$ ) of this pointing is ideal to look for variability on similar time scales as those investigated by G02. Using the BeppoSAX MECS counts in the 2-10 keV energy range $^{4}$ we do not find evidence of variability: the $\chi^{2}$ test, applied to the binned light curve $(\Delta T=5700 \mathrm{~s})$, gives a probability of $50 \%$ that the data are consistent with a constant flux during the BeppoSAX observation. It is worth noting that this result does not exclude variability on different time scales and/or irregular variability.

Finally, the XMM-Newton and BeppoSAX observations are not simultaneous but the MECS and XMM-Newton 2-10 keV fluxes, derived assuming a simple power-law (PL) model,

\footnotetext{
${ }^{4}$ The BeppoSAX PSF does not allow us to extract a nuclear light curve, as done by G02 with the Chandra data, but we already know that the soft starburst contribution is not significant above $2 \mathrm{keV}$.
}

are comparable within the relative uncertainties; thus a statistically significant flux difference is not found between the two data sets.

\section{Spectral analysis}

The XMM-Newton and BeppoSAX data have been analyzed using standard software packages (FTOOLS 5.2, XSPEC 11.2). All models discussed here have been filtered for the Galactic absorbing column density along the line of sight $\left(N_{\mathrm{H}}=\right.$ $1.3 \times 10^{20} \mathrm{~cm}^{-2}$ Dickey \& Lockman 1990). Unless otherwise stated, errors will be given at the $90 \%$ confidence level for one interesting parameter $\left(\Delta \chi^{2}=2.71\right)$ and the line(s) energy will be given in the source rest frame. The thermal emission has been described with the MEKAL model in XSPEC (Mewe et al. 1985). Abundances were kept solar; keeping them free did not substantially change the results. Since MKN 231 does not show significant flux variation we have first derived the cross-calibration between MOS, pn and MECS and then, by assuming that the cross-calibration factor between MECS and PDS ranges from 0.7 to 0.95 (see Fiore et al. 1999), we have tied together the XMM-Newton data and the PDS data.

From previous X-ray observations, performed with ASCA and Chandra, we know that the broad-band $(0.2-10 \mathrm{keV})$ spectrum of MKN 231 appears to be more complex than a single power-law model. In particular the Chandra data presented by G02 show that the circumnuclear starburst thermal emission, which extends up to $\sim 25^{\prime \prime}$ from the nucleus, dominates the spectrum at $E<2 \mathrm{keV}$. Due to the PSF of XMM-Newton and BeppoSAX detectors the extracted counts in the low energy domain are therefore "contaminated" by the circumnuclear starburst. In contrast the hard $(E>2 \mathrm{keV}) \mathrm{X}$-ray emission should be less affected by the starburst activity and more suitable for investigating AGN related emission(s). For these reasons we have decided to focus our attention first on the 2-50 keV spectrum, and for simplicity (since we have enough statistics) to use only the pn and the PDS data. In a second step we have also considered the $0.5-2 \mathrm{keV}$ source counts as well as the data from all the other detectors (MECS, MOS1 and MOS2).

\subsection{Fitting the hard (2-50 keV) X-ray energy band: pn + PDS data}

In agreement with previous analysis (Iwasasawa 1999; Turner 1999; MR00 and G02) when we fit the $2-10 \mathrm{keV}$ spectrum of MKN 231 with a single unabsorbed or absorbed PL model, we find a photon index $\left(\Gamma=0.67 \pm 0.11\right.$ and $\Gamma=0.92_{-0.23}^{+0.28}$, respectively) which is unusually low if compared with the typical photon index of radio-quiet QSO ( $\Gamma$ ranges from 1.7 to 2.3, Reeves \& Turner 2000; George et al. 2000). Furthermore the fit leaves positive residuals around $6.4 \mathrm{keV}$, strongly suggesting the presence of the iron emission line(s). The best fit energy position of this line is $6.40_{-0.11}^{+0.15} \mathrm{keV}$ with an observed $E W=$ $300 \pm 130 \mathrm{eV}$, in agreement with the ASCA data discussed by MR00 (in this first modelling the Fe line was constrained to be unresolved). The flatness of the observed X-ray spectrum is suggested also by the high energy $(E=10-50 \mathrm{keV})$ 
PDS data: in fact if we constrain the photon index to lie in the range 1.7-2.3, the PDS data are underestimated by at least a factor of 4 . These results suggests: a) the presence of a heavily absorbed component and b) that below $10 \mathrm{keV}$ we are probably seeing only the reprocessed radiation (through reflection and/or scattering, see Matt et al. 1991). In the following we investigate in turn two extreme possibilities: a reflection-dominated and a scattering-dominated scenario.

\subsubsection{The reflection-dominated scenario}

In the reflection-dominated scenario the bulk of the $2-10 \mathrm{keV}$ emission derives from Compton reflection from optically thick material. When we consider reflection from neutral matter a strong (i.e. $E W$ as high as a few keV; Matt et al. 1996) Fe emission line is also expected, this line being produced by the same medium which reflects the primary continuum. The lack of a strong $\mathrm{Fe}$ emission line seems then to argue against a reflection-dominated scenario. However, the intensity and the energy centroid of the Fe line depends on the ionization state of the medium (Matt et al. 1993; Ross et al. 1999); in particular, the intensity decreases, compared to reflection from a neutral medium, when the ionization parameter $(\xi)$ is between 100 and 300, due to the resonant trapping opacity (Zycki \& Czerny 1994).

\section{a) Neutral medium}

A pure reflection-dominated continuum, where the reflecting medium is neutral, cannot reproduce the $2-50 \mathrm{keV}$ spectral properties of MKN 231, leaving strong positive residuals above $7 \mathrm{keV}$. Furthermore this model would require a $\mathrm{Fe}$ emission line at $6.4 \mathrm{keV}$ stronger than that measured. As previously noted by MR00, a prominent scattered component is needed to reproduce the observed continuum and to dilute the $\mathrm{Fe}$ line. We then tested the pure reflection plus scattering model using a PL plus a pure reflected continuum $\left(\mathrm{PEXRAV}^{5}\right.$ model in XSPEC; Magdziarz \& Zdziarski 1995) plus an unresolved Gaussian line. The best fit photon index $(\Gamma \sim 1.3)$ is still low if compared with the typical photon index of radio-quiet QSOs. Constraining the photon index to lie in the range 1.7-2.3 we cannot find an acceptable fit. The results are summarized in Table 1, model A and model B. These models do not consistently explain the low observed $E W$ of the line and the flatness of the spectrum for an intrinsic standard AGN emission.

\section{b) Ionized medium}

An alternative explanation for the observed low $E W$ of the $\mathrm{Fe}$ line invokes the ionization state of the reflecting medium.

\footnotetext{
${ }^{5}$ Given the present statistics above $20 \mathrm{keV}$ we fixed $\cos (i)=0.45$, $Z=Z_{\odot}$ and $E_{\mathrm{c}}=200 \mathrm{keV}$ and allowed only the photon index and the normalization to vary.
}

Since the reflected continuum from mildly ionized material predicts the presence of structured features in the low-energy domain (see e.g. Ross \& Fabian 1993) we are forced to consider also the pn counts below $2 \mathrm{keV}$. On the other hand, below $2 \mathrm{keV}$ we also have to take into account the contribution from the circumnuclear starburst.

Thus, we have first tested a 3-component model composed of: a) thermal emission to take into account the starburst contribution at low energies; b) a pure reflected component from mildly ionized material (PEXRIV ${ }^{6}$ model in XSPEC; Magdziarz \& Zdziarski 1995) and c) a Gaussian line.

This model gives a poor representation of the broad band $\left(\mathrm{pn}+\mathrm{PDS}\right.$ data) properties of MKN $231\left(\chi^{2} / v=102.6 / 65\right.$; see Table 1 model $\mathrm{C}$ ), leaving strong positive residuals at energies above $7 \mathrm{keV}$ and underestimating by a factor 5 the counts in the PDS energy range; all these features strongly suggest the presence of a heavily absorbed $\left(N_{\mathrm{H}} \gtrsim 10^{24} \mathrm{~cm}^{-2}\right)$ component. We have thus added a highly absorbed PL component (described by the PLCABS ${ }^{7}$ model in XSPEC; Yaqoob 1997) whose photon index has been tied to the photon index of the reflected component from ionized material.

This model gives a better broad band description of the spectrum of MKN 231 (see Table 1 model D), but it requires a very steep intrinsic photon index $(\Gamma=2.95 \pm 0.07)$.

Finally, given that MKN 231 has a strong circumnuclear starburst (see introduction), and given the size of the source counts extraction radius, we expect a contribution from X-ray binaries, and in particular from High-Mass X-ray binaries (Franceschini et al. 2003; Persic et al. in preparation). This latter component has been described assuming an absorbed cutoff-power law model of the form $E^{-\Gamma} \exp ^{-h v / k T}$ with photon index $\Gamma \sim 1.1$ and cutoff energy $k T \sim 10 \mathrm{keV}$ (cf. Persic \& Raphaeli 2002).

This final model gives a good description of the X-ray spectra of MKN 231. In Fig. 1 (left panel) we show the data and the residuals while the best fit spectral parameters are reported in Table 2 (model A).

The line energy position is $6.38 \pm 0.19$ at $90 \%$ confidence level, in agreement with the value $(6.5 \mathrm{keV})$ expected for this range of ionization of the mirror. The $E W$ of the line is $\sim 200 \mathrm{eV}$ with respect to the reflected component, which is consistent with being produced by a mildly ionized mirror (Ross et al. 1999). By allowing the line width to vary we obtain $\sigma \sim 0.1 \mathrm{keV}$, consistent with the XMM-Newton spectral resolution. Thus at the spectral resolution of XMM-Newton the detected Fe line is unresolved. No statistical difference for the best fit parameters reported in Table 2 is found if the line width is allowed to vary.

In conclusion, a pure reflection-dominated continuum from neutral material cannot consistently explain the low

\footnotetext{
${ }^{6}$ Given the present statistics above $20 \mathrm{keV}$ we fixed $\cos (i)=0.45$, $Z=Z_{\odot}$ and $E_{\mathrm{c}}=200 \mathrm{keV}$ and allowed only the photon index and the normalization to vary. The ionization parameter $\xi$ was constrained to lie in the range 100-300.

${ }^{7}$ Given that the intrinsic $N_{\mathrm{H}}$ is expected to be high $\left(\sim 10^{24} \mathrm{~cm}^{-2}\right)$ we have used this model, which takes into account also the Compton scattering.
} 
Table 1. Pn plus PDS data: rest-frame results from the basic reflection-dominated models discussed in Sect. 4.1.1.

\begin{tabular}{lll|ll}
\hline \hline \multirow{2}{*}{ Model } & & \multicolumn{2}{c}{ Fe line } \\
& Parameter & Value & Parameter & Value \\
\hline \multirow{2}{*}{ A) refl PL+Fe line } & $\Gamma^{a}$ & $2.65_{-0.19}^{+0.19}$ & $E_{\mathrm{K} \alpha}$ & $6.41_{-0.18}^{+0.22} \mathrm{keV}$ \\
& $N_{\mathrm{H}}^{b}$ & $/$ & $E W$ & $<0.24 \mathrm{keV}$ \\
& $R$ & $1.3_{-0.1}^{*}$ & & \\
& $N_{\mathrm{H}}^{c}$ & $/$ & $\chi^{2} /$ d.o.f. & $35.61 / 29$ \\
\hline \multirow{3}{*}{ B) refl PL+Fe line +scatt PL } & $\Gamma^{a}$ & $1.32_{-0.59}^{+0.37}$ & $E_{\mathrm{K} \alpha}$ & $6.42_{-0.16}^{+0.22} \mathrm{keV}$ \\
& $N_{\mathrm{H}}^{b}$ & $/$ & $E W$ & $0.28_{-0.20}^{+0.30} \mathrm{keV}$ \\
& $R$ & $1.01_{-1.01}^{+5400}$ & $\sigma$ & $0.16_{-0.16}^{+0.30} \mathrm{keV}$ \\
& $N_{\mathrm{H}}^{c}$ & $2.14_{-1.25}^{+1.35}$ & $\chi^{2} /$ d.o.f. & $19.6 / 26$ \\
\hline & $\Gamma^{a}$ & $2.84_{-0.12}^{+0.13}$ & $E_{\mathrm{K} \alpha}$ & $6.40_{-0.19}^{+0.24} \mathrm{keV}$ \\
& $N_{\mathrm{H}}^{b}$ & $0.19_{-0.10}^{+0.11}$ & $E W$ & $0.18_{-0.14}^{+0.18} \mathrm{keV}$ \\
& $R$ & $0.94_{-0.16}^{+1150}$ & $\sigma$ & $0.10_{-0.10}^{+0.26} \mathrm{keV}$ \\
& $N_{\mathrm{H}}^{c}$ & $/$ & $\chi^{2} /$ d.o.f. & $102.6 / 65$ \\
\hline & $\Gamma^{a}$ & $2.95_{-0.16}^{+0.15}$ & $E_{\mathrm{K} \alpha}$ & $6.39_{-0.20}^{+0.18} \mathrm{keV}$ \\
& $N_{\mathrm{H}}^{b}$ & $0.24_{-0.10}^{+0.15}$ & $E W$ & $0.18_{-0.15}^{+0.17} \mathrm{keV}$ \\
& $R$ & $1.04_{-0.15}^{+1042}$ & $\sigma$ & $0.10_{-0.10}^{+0.25} \mathrm{keV}$ \\
& $N_{\mathrm{H}}^{c}$ & $272.6_{-68.0}^{+99.9}$ & $\chi^{2} /$ d.o.f. & $89.0 / 61$ \\
\hline
\end{tabular}

For model A and model B we have considered the $2-50 \mathrm{keV}$ data, while for model $\mathrm{C}$ and $\mathrm{D}$ we have considered the $0.2-50 \mathrm{keV}$ data. For model $\mathrm{C}$ and $\mathrm{D}$ the ionization parameter has been constrained to lie within the range $100-300 \mathrm{erg} \mathrm{cm} / \mathrm{s}$.

${ }^{a}$ Intrinsic photon index of the reflected and scattered component; in model D the photon indices of the transmitted and reflected component have been tied together.

${ }^{b}$ Absorption of the reflected component in units of $10^{22} \mathrm{~cm}^{-2}$.

${ }^{c}$ Absorption of the scattered (transmitted) component in units of $10^{22} \mathrm{~cm}^{-2}$.

* Unconstrained parameter.

Fe emission line $E W$ and the flatness of the spectrum. A viable solution is the pure reflection continuum from slightly ionized material combined with a highly absorbed component. Adding to this "AGN baseline model" the spectral components which are likely associated to the starburst (a soft thermal component and a PL component associated to the High Mass X-ray Binaries) we found a value of $\sim 2.5$ for the AGN intrinsic photon index. This steep intrinsic photon index is not so striking if we consider that MKN 231 shares many spectral characteristic of NLS1s (i.e. weak $\left[\mathrm{O}_{\mathrm{III}}\right]$, relatively narrow Balmer emission lines and strong optical $\mathrm{Fe}_{\text {II }}$ emission; Mathur 2000; Brandt \& Gallagher 2000). According to this model, hereafter called the "AGN reflection-dominated" model, the intrinsic 2-10 keV luminosity of the AGN powering MKN 231 is $L_{(2-10)} \sim 2 \times 10^{44} \mathrm{erg} \mathrm{s}^{-1}$, in agreement with the value predicted by MR00 based on ASCA data.

\subsubsection{The scattering-dominated scenario}

Another possible explanation for the hard MKN 231 X-ray continuum flatness and the lack of a strong $\mathrm{Fe}$ emission line is a scattering-dominated scenario in which we see the AGN mainly through scattered emission (see G02 modeling of the Chandra data). In this context we assume that the Fe emission line is produced by transmission and is diluted from the scattered component.

To test this scenario we have used a 3-component model composed of: a) a highly absorbed PL representing the intrinsic AGN emission transmitted through the Compton-thick screen; b) a PL having the same photon index as the transmitted component, representing the scattered AGN emission and c) a Gaussian line. This model gives a fairly good description of the data $\left(\chi^{2} / v=20.47 / 27\right)$ and, as shown in Fig. 1 (right panel), no strong residuals are present.

The intrinsic AGN emission is described by a PL model having a photon index of $\Gamma=1.69_{-0.70}^{+0.49}$ which is filtered by an intrinsic absorption column density of $N_{\mathrm{H}}=1.4_{-0.5}^{+0.9} \times$ $10^{24} \mathrm{~cm}^{-2}$. The energy position of the Gaussian line best fit value is $E=6.39_{-0.12}^{+0.13} \mathrm{keV}$, which is consistent with the neutral $\mathrm{Fe}-\mathrm{K} \alpha$ line. Allowing the line width to vary we obtain $\sigma=0.2 \pm 0.18 \mathrm{keV}$ (we note however that if we allow the line width to vary the fit does not improve significantly). The Fe line $E W$ is $\sim 260 \mathrm{eV}$ with respect to the observed continuum and $\sim 1.2 \mathrm{keV}$ with respect to the absorbed PL. This latter value is consistent with that expected to be produced by transmission in the same medium that absorbs the primary 

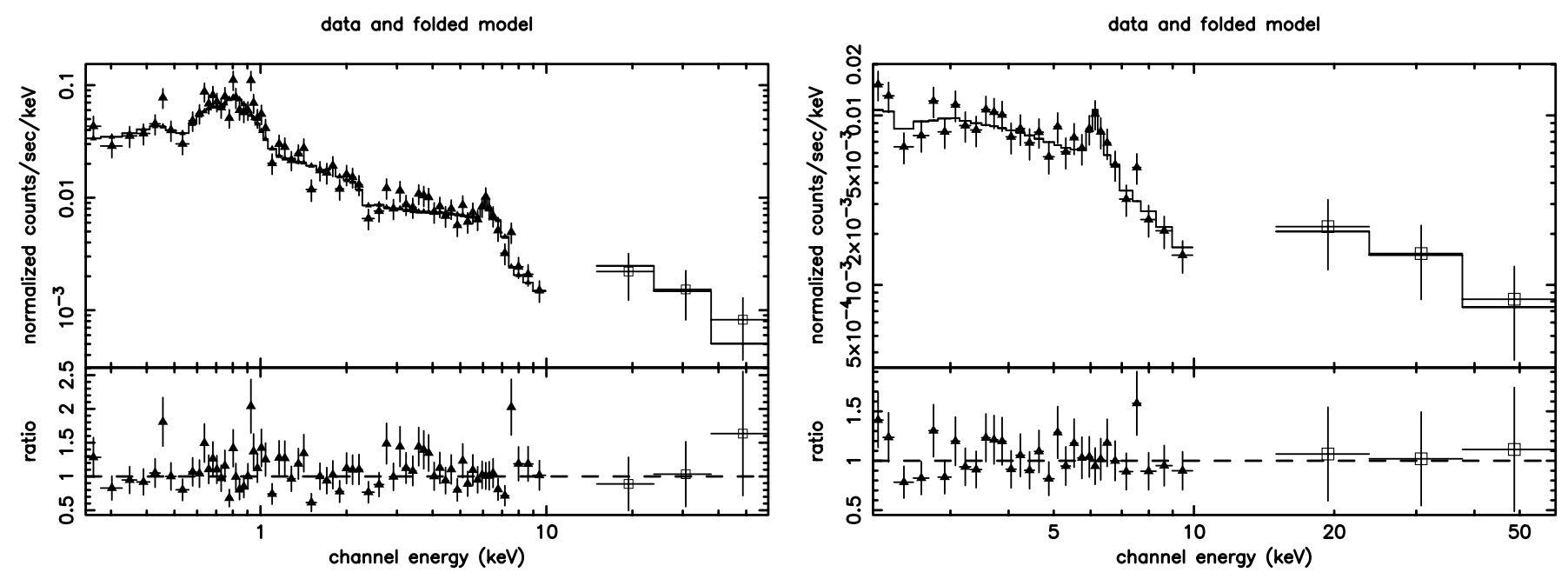

Fig. 1. Left panel: $0.2-50 \mathrm{keV}$ pn (filled triangles) plus PDS (empty squares) data and ratio between data and the best fit reflection model (model A in Table 2). Right panel: 2-50 keV pn (filled triangles) plus PDS (empty squares) data and ratio between data and the scattering dominated model discussed in Sect. 4.1.2.

Table 2. Broad-band best fit models and parameters: model A refers to the reflection-dominated model, while model B refers to the scatteringdominated model. Both models include the starburst components.

\begin{tabular}{|c|c|c|c|c|}
\hline \multicolumn{3}{|c|}{ Starburst } & \multicolumn{2}{|c|}{ AGN } \\
\hline & Parameter & Value & Parameter & Value \\
\hline \multirow{5}{*}{ Model A } & $k T$ & $0.66_{-0.05}^{+0.05} \mathrm{keV}$ & $\Gamma$ & $2.48_{-0.11}^{+0.20}$ \\
\hline & / & & $N_{\mathrm{H}}^{b}$ & $0.20_{-0.04}^{+0.08}$ \\
\hline & $\Gamma_{\mathrm{BIN}}$ & $1.1^{\star}$ & $N_{\mathrm{H}}^{c}$ & $264.6_{-85.1}^{+173.2}$ \\
\hline & $N_{\mathrm{H}}^{a}$ & $4.22_{-4.22}^{+16.9}$ & $E_{\mathrm{K} \alpha}$ & $6.38_{-0.17}^{+0.19} \mathrm{keV}$ \\
\hline & & & $E W$ & $0.20_{-0.16}^{+0.16} \mathrm{keV}$ \\
\hline \multirow{5}{*}{ Model B } & $k T_{1}$ & $0.35_{-0.05}^{+0.09} \mathrm{keV}$ & $\Gamma$ & $1.83_{-0.69}^{+0.12}$ \\
\hline & $k T_{2}$ & $0.91_{-0.10}^{+0.15} \mathrm{keV}$ & $N_{\mathrm{H}}^{b}$ & $12.16_{-2.03}^{+2.67}$ \\
\hline & $\Gamma_{\mathrm{BIN}}$ & $1.1^{\star}$ & $N_{\mathrm{H}}^{c}$ & $177.5_{-110.0}^{+105.8}$ \\
\hline & $N_{\mathrm{H}}^{a}$ & $1.7_{-1.7}^{+6.4}$ & $E_{\mathrm{K} \alpha}$ & $6.39_{-0.14}^{+0.15} \mathrm{keV}$ \\
\hline & & & $E W$ & $0.29_{-0.18}^{+0.18} \mathrm{keV}$ \\
\hline
\end{tabular}

${ }^{a}$ Absorption of the HMXBs component in units of $10^{20} \mathrm{~cm}^{-2}$.

${ }^{b}$ Absorption of the reflected/scattered component (model A/model B) in units of $10^{22} \mathrm{~cm}^{-2}$.

${ }^{c}$ Absorption of the transmitted component in units of $10^{22} \mathrm{~cm}^{-2}$;

the symbol * indicate that the parameter has been kept fixed.

nuclear emission (Leahy \& Creighton 1993). The scattered component is also absorbed by an intrinsic absorption column density of $N_{\mathrm{H}}=4.1_{-3.2}^{+1.8} \times 10^{22} \mathrm{~cm}^{-2}$.

In the $2-10 \mathrm{keV}$ band the observed flux is $7 \times$ $10^{-13} \mathrm{ergs} \mathrm{cm}^{-2} \mathrm{~s}^{-1}$ and the intrinsic $2-10 \mathrm{keV}$ luminosity, de-absorbed for the intrinsic $N_{\mathrm{H}}$, is $L_{(2-10)}=4 \times 10^{43} \mathrm{erg} \mathrm{s}^{-1}$.

In summary this model provides another possible explanation for the flat MKN 231 observed 2-50 keV continuum and its line properties.

Furthermore, although not well constrained, the best fit photon index is now fully consistent with the range usually found in AGN ( $\Gamma \simeq 1.7-2.3$ ). In the following we will refer to this model as the "AGN scattering-dominated" model.

\subsection{The broad band (0.5-50 keV) X-ray spectral properties of MKN 231}

In the previous section, using the pn and the PDS data, we found that two competitive models (reflection-dominated and scattering-dominated) can describe the MKN 231 AGN emission. In this section these two models will be used as starting points to study the broad band $(0.5-50 \mathrm{keV}) \mathrm{X}$-ray spectral properties of MKN 231. Low energy data $(E<2 \mathrm{keV})$ and all the detectors MOS1, MOS2, pn, MECS together with the PDS data will be used.

In the ionized reflection model case we found that the model derived for the pn and the PDS data (which already 
Current model
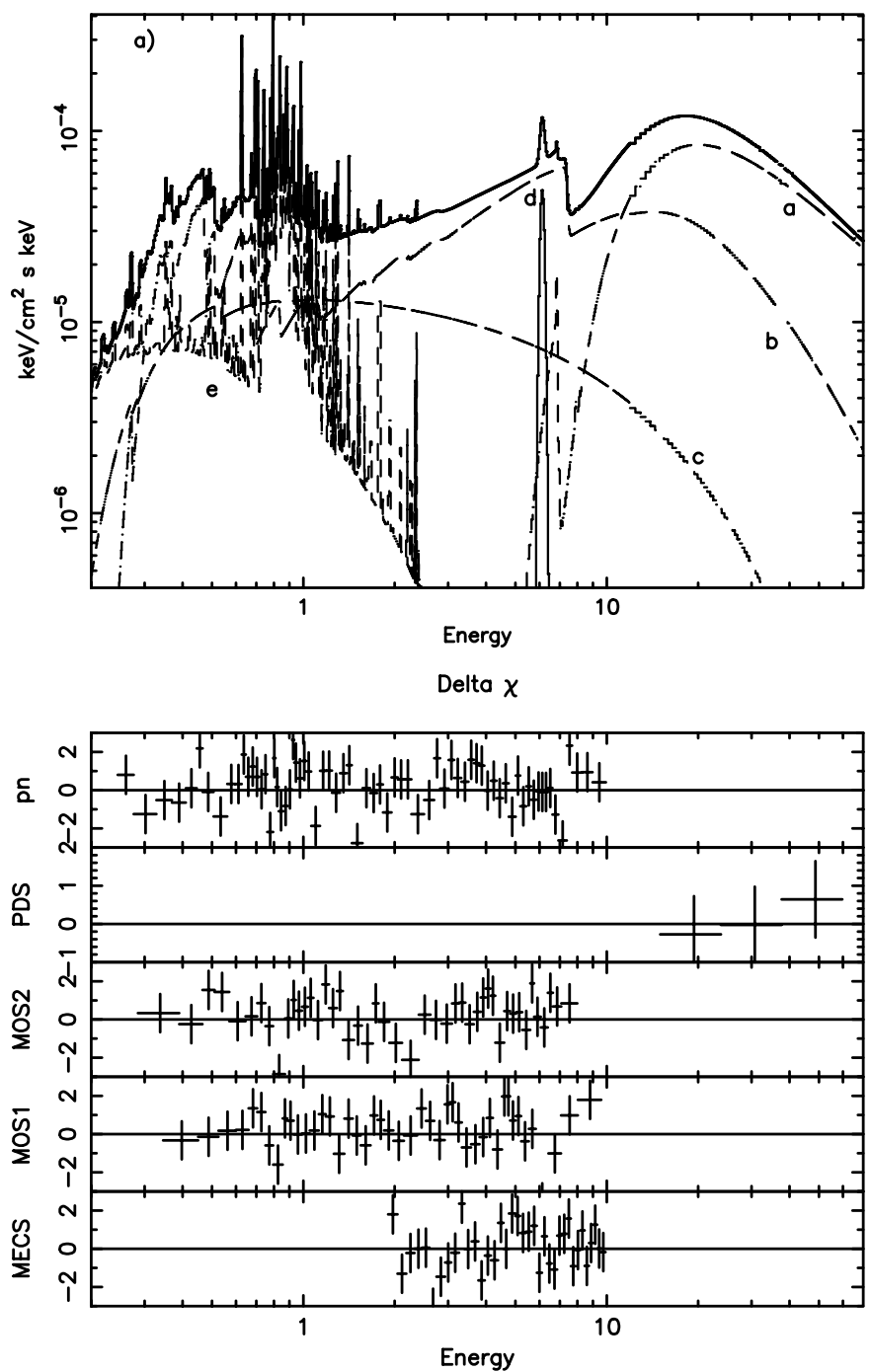

Current model
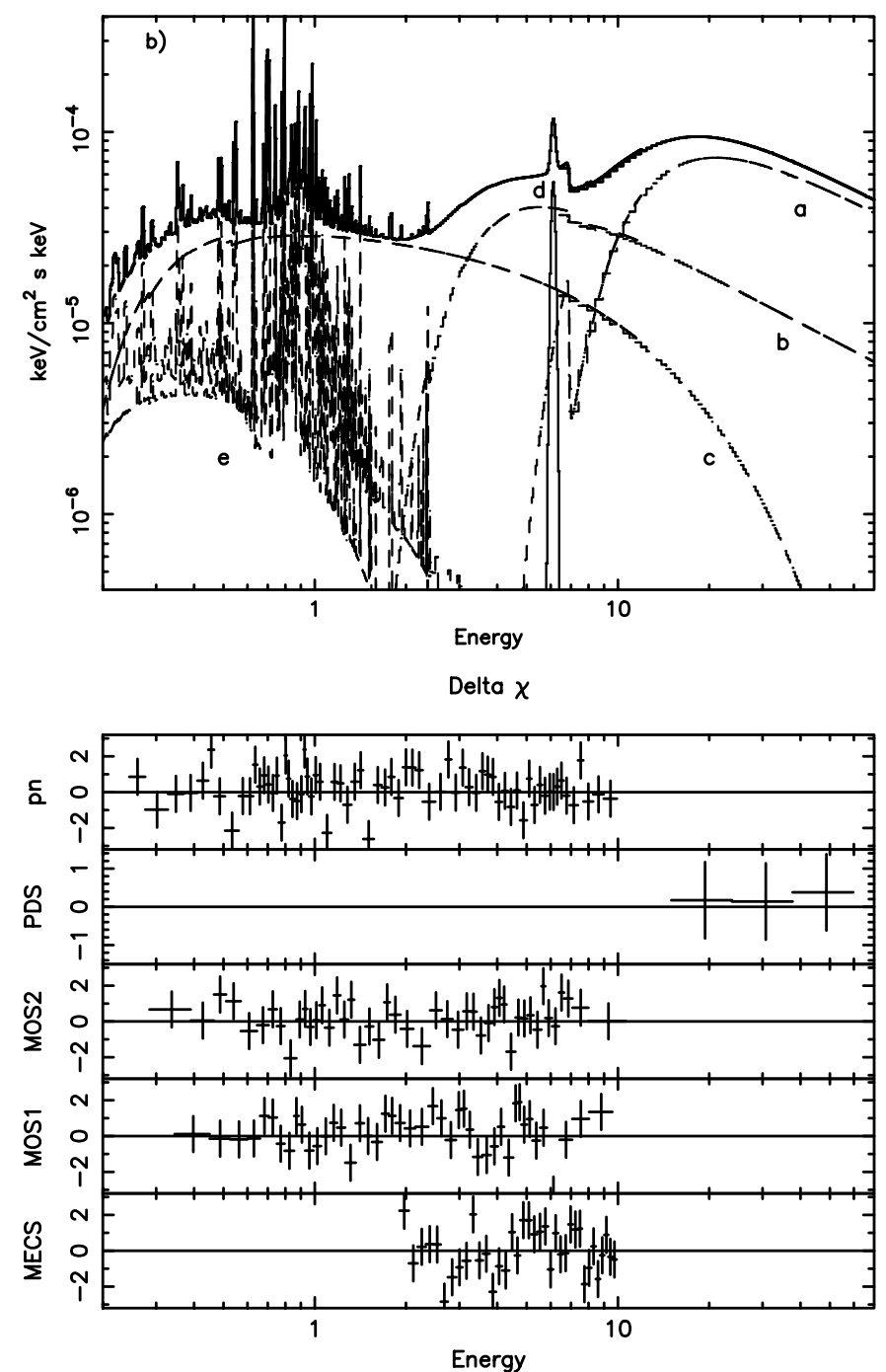

Fig. 2. $0.2-50 \mathrm{keV}$ data coverage. Upper panels: best-fit models for the reflected-dominated (left panel) and scattering-dominated scenario (right panel). The spectral components are: a) a highly absorbed PL AGN component; b) left panel: a pure reflection AGN component from slightly ionized material or right panel: scattered AGN component; c) a cutoff PL component associated with the binaries in the starburst; d) a narrow Gaussian line at $6.39 \mathrm{keV}$; and e) a thermal emission component associated with the starburst. Lower panels: residuals for the different detectors (from up to down: pn, PDS, MOS2, MOS1, MECS).

include the spectral components expected from the starburst, see Sect. 4.1.1) is able to reproduce all data with no strong differences for the previously determined best-fit parameters. In Fig. 2 (left panels) we show the model and the residuals for all the detectors, while the best fit spectral parameters are reported in Table 2 (model A). Within the framework of this modelling the intrinsic luminosity of the AGN powering MKN 231 is: $L_{(0.5-2)}=3.3 \times 10^{44} \mathrm{erg} \mathrm{s}^{-1}$ and $L_{(2-10)}=2 \times 10^{44} \mathrm{erg} \mathrm{s}^{-1}$ (see Table 3).

In the scattering-dominated scenario case the residuals below $2 \mathrm{keV}$ are indicative of thermal emission. Indeed, two thermal components are needed to reproduce the soft X-ray emission of MKN $231\left(k T \sim 0.35_{-0.05}^{+0.09} \mathrm{keV}\right.$ and $k T \sim$ $\left.0.91_{-0.10}^{+0.15} \mathrm{keV}\right)$. For the HMXBs emission we have added an absorbed cutoff PL component, following what already done in Sect. 4.1.1; moreover, this component is needed to reproduce the continuum detected in the $1-3 \mathrm{keV}$ range. The best-fit spectral parameters derived with this broad-band, multi-component modelling are reported in Table 2 (model B; $\left.\chi^{2} / v=212.9 / 187\right)$. Note that the best-fit photon index for the AGN component is now $\Gamma=1.83_{-0.69}^{+0.12}$. The derived intrinsic $2-10 \mathrm{keV}$ luminosity for the $\mathrm{AGN}$ is $L_{(2-10)}=4.5 \times$ $10^{43} \mathrm{erg} \mathrm{s}^{-1}$ A summary view of the model is reported in Fig. 2 (right panel).

\section{Discussion}

\subsection{The AGN component in MKN 231}

The AGN spectral behavior can be described with two models which differ mainly at $E \lesssim 8 \mathrm{keV}$. Both these models require a heavily absorbed component to account for the X-ray emission above $10 \mathrm{keV}$ detected with the PDS. The photon 
Table 3. Observed X-ray Fluxes and intrinsic X-ray luminosities derived in the two competing scenarios.

\begin{tabular}{|c|c|c|c|c|c|c|c|}
\hline \multirow[b]{2}{*}{ Model } & \multicolumn{3}{|c|}{$\begin{array}{l}\text { Flux }(0.5-10) \mathrm{keV} \\
10^{-13} \mathrm{ergs} \mathrm{cm}^{-2} \mathrm{~s}^{-1}\end{array}$} & \multicolumn{2}{|c|}{$\begin{array}{c}L(0.5-2) \mathrm{keV} \\
10^{43} \mathrm{erg} \mathrm{s}^{-1}\end{array}$} & \multicolumn{2}{|c|}{$\begin{array}{c}L(2-10) \mathrm{keV} \\
10^{43} \mathrm{erg} \mathrm{s}^{-1}\end{array}$} \\
\hline & Total & AGN & Starburst & AGN & Starburst & $\overline{A G N}$ & Starburst \\
\hline A & 7.9 & 6.3 & 1.6 & 33 & 0.06 & 20 & 0.07 \\
\hline B & 8.2 & 5.0 & 3.2 & 2.9 & 0.09 & 4.5 & 0.16 \\
\hline
\end{tabular}

Model A refers to reflection-dominated model.

Model B refers to scattering-dominated model.

indices derived with these models, although not well constrained, are consistent with the typical values of AGNs. Furthermore the PDS data have proven, for the first time, the presence of a thick column density screen $\left(N_{\mathrm{H}} \sim 2 \times\right.$ $10^{24} \mathrm{~cm}^{-2}$ ) which blocks the primary X-ray emission. The 2$10 \mathrm{keV}$ observed spectrum could be the result of reflection (reflection-dominated scenario) or of scattering (scatteringdominated scenario) which emerges from the Compton-thick screen. Clearly the real physical situation could be an intermediate one where we see both components. Since the complexity of the $2-10 \mathrm{keV}$ continuum prevents us from distinguishing, on a statistical basis, between these two models, we have checked if the physical parameters derived in both scenarios are in agreement with what is known about ULIRGs, starburst galaxies and in particular with the broad-band properties of MKN 231.

We identify the optically thick absorber with the BAL outflows which originate close to the central source. The ionized matter, which scatters or reflects the primary emission, could be the inner part of this outflow, or "shielding gas" (Murray et al. 1995) in agreement with the scenario proposed by G02. In both models proposed to take into account the AGN emission the scattered/reflected components are absorbed. This latter absorbing medium could be identified with the starburst regions (see e.g. Levenson et al. 2001) or with a different line of sight through the BAL wind.

Beside the flat shape of the observed MKN 231 X-ray continuum, the other topic that emerged, since the first hard X-ray observations, is the low $2-10 \mathrm{keV}$ luminosity. If we assume that the far-infrared emission of MKN 231 is mainly powered by AGN activity then the intrinsic X-ray luminosity should be $\sim 10^{45} \mathrm{erg} \mathrm{s}^{-1}$ (Risaliti et al. 2000). In both models the intrinsic 2-10 keV X-ray luminosity can be measured from the absorbed component and is $\sim 1_{-0.5}^{+1.0} \times 10^{44} \mathrm{erg} \mathrm{s}^{-1}$. Although the balance between the X-ray and bolometric luminosity is derived assuming a standard type $1 \mathrm{AGN}$, this estimate suggests that the contribution of the starburst activity to the FIR luminosity could be significantly higher than that of the AGN.

\subsection{The starburst component in MKN 231}

In both AGN models proposed we found a strong soft X-ray component which we describe with thermal emission(s) associated to the starburst activity. The derived temperature(s) are in agreement with the results obtained for the extended emission by G02 using Chandra data $(k T \sim 0.3 \mathrm{keV}$ and $k T \sim 1.1 \mathrm{keV})$ and consistent with that measured in other well known bonafide luminous starburst galaxies.

The energetics of this thermal component(s) $\left(L_{(0.5-2)}=\right.$ $6-9 \times 10^{41} \mathrm{erg} \mathrm{s}^{-1}$ depending on the AGN modelling) is comparable with what has been previously found for ULIRGs (Franceschini et al. 2003; Ptak et al. 2003). The measured luminosities are in agreement with the one estimated using ROSAT and ASCA data (Iwasawa 1999; Turner 1999), but about a factor 4-6 higher than that found by G02 using Chandra data $\left(L_{(0.5-2)}=10^{41.2} \mathrm{erg} \mathrm{s}^{-1}\right)$.

For the hard X-ray emission, which we attribute to the $\mathrm{HMXB}$, we found that $L_{(2-10)}$ ranges from $6.9 \times 10^{41} \mathrm{erg} \mathrm{s}^{-1}$ to $1.6 \times 10^{42} \mathrm{erg} \mathrm{s}^{-1}$ (in the reflection and in the scatteringdominated scenario respectively). This spectral component is expected in a source like MKN 231 with a strong nuclear starburst and has to be taken into account in the spectral modelling. We have checked if the X-ray luminosity of the cutoff $\mathrm{PL}$ component is in agreement with the results obtained by Franceschini et al. (2003) for a sample of ULIRGs observed with XMM-Newton. These observations have shown that for all the starburst dominated ULIRGs the hard X-ray luminosity of the X-ray binaries clearly correlates with the FIR luminosity.

In Fig. 3 we show the result of the comparisons between the $\mathrm{X}$-ray emission due to starburst and to AGN activity and $L_{\mathrm{FIR}}$ for the 10 ULIRGs observed with XMM-Newton; in these plots we have assumed that MKN 231 starburst activity contributes for $\sim 50 \%$ to the $L_{\mathrm{FIR}}$.

The range of the 2-10 keV HMXB luminosity derived with the two different AGN models, clearly locates MKN 231 in the region occupied by the starburst-dominated ULIRGs. This result gives confidence that the power-law component originates from the population of HMXB expected to be present in the circumnuclear starburst region of MKN 231.

We have also compared the star-formation rate (SFR) predicted from the 2-10 X-ray emission with that derived by the FIR luminosity. To do that we have taken into account that the latter contributes to the $2-10 \mathrm{keV}$ emission mainly through the HMXB emission in case of intense and recent star-forming activity (Franceschini et al. 2003; Persic et al. in prep.) ${ }^{8}$. This assumption is consistent with a significant contribution of the

\footnotetext{
8 The relation between HMXB luminosity and SFR has been recently discussed in the literature (i.e. Grimm et al. 2003; Gilfanov et al. 2004; Ranalli et al. 2003).
} 

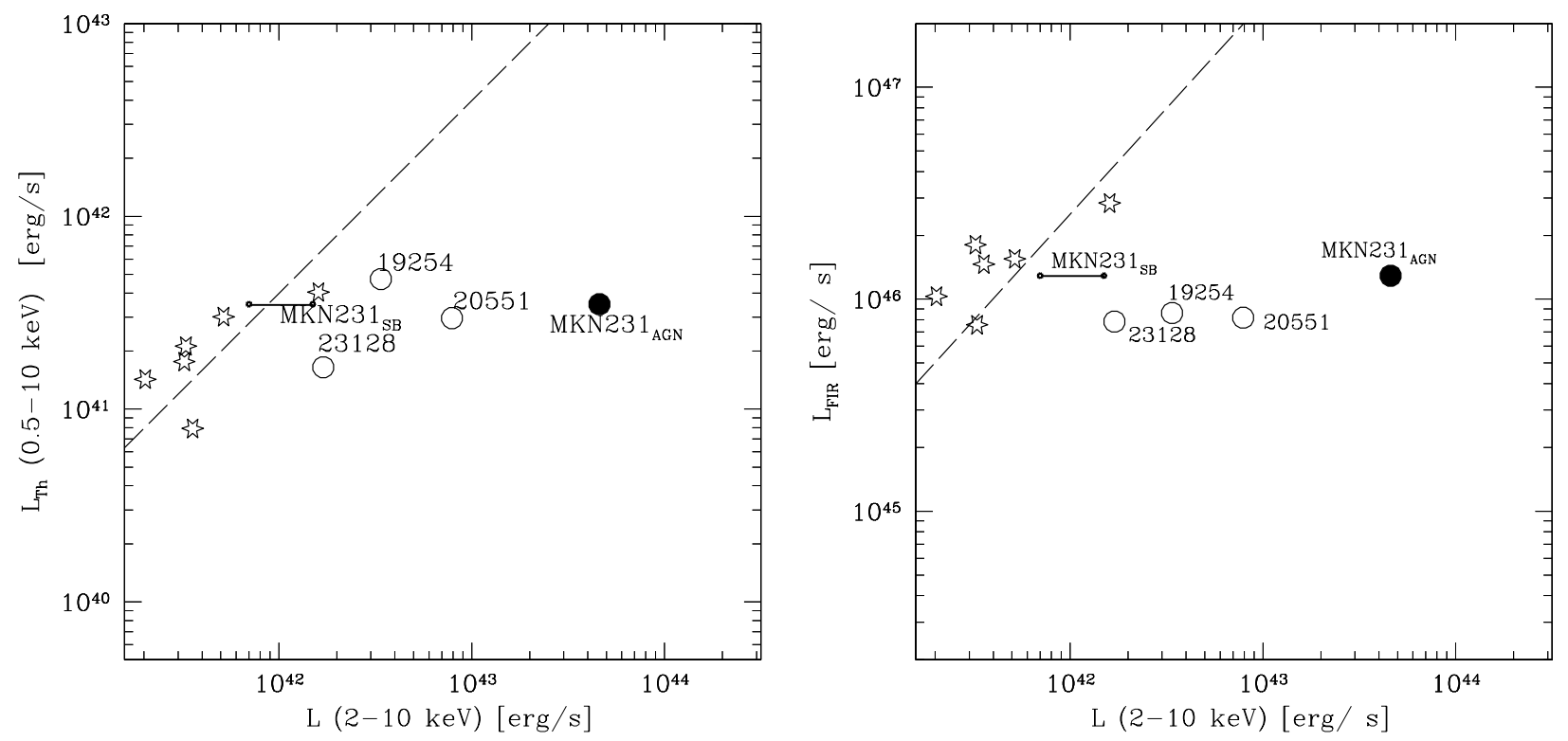

Fig. 3. Luminosity-luminosity plots of various emission components identified in the XMM-Newton and BeppoSAX spectrum of MKN 231 compared with the ULIRGs in Franceschini et al. (2003). Starred symbols refer to sources classified as dominated by starburst, open circles to AGN-dominated sources. For MKN 231 we report (black solid line) the range of the hard X-ray luminosity of the binary components and the lower limit of the AGN (filled circle) hard X-ray luminosity; we have also assumed that the starburst activity in MKN 231 contributes to $50 \%$ of $L_{\mathrm{FIR}}$. Left panel: luminosity-luminosity plot of the thermal versus PL components. Right panels: $L_{\mathrm{FIR}}$ against the luminosities of the PL components. Dashed lines indicate proportionality relations.

starburst activity to the $L_{\mathrm{FIR}}$. Our result is in fair agreement with the results obtained with the modelling of the $1-1000 \mu \mathrm{m}$ Spectral Energy Distribution by Farrah et al. (2003).

Considering the heavily obscured PL detected with the PDS and the above starburst scenario MKN 231 no longer appears as an unusually X-ray faint AGN. In conclusion, XMM-Newton and BeppoSAX observations suggest that starburst activity strongly contributes to the $L_{\mathrm{FIR}}$ of MKN 231, in agreement with multiwavelength analysis.

\section{Summary}

By combining the XMM-Newton spectral resolution and throughput with the high-energy bandpass of BeppoSAX we have investigated in deeper detail than previously possible the broad-band $(0.2-50 \mathrm{keV}) \mathrm{X}$-ray spectral properties of MKN 231

We confirm that MKN 231 is a complex source whose $\mathrm{X}$-ray spectrum has clear signatures of both a highly obscured AGN and strong starburst activity.

The detection, in the PDS energy range, of a highly absorbed $\left(N_{\mathrm{H}} \sim 2 \times 10^{24} \mathrm{~cm}^{-2}\right)$ component for the first time makes it possible to measure the AGN intrinsic $2-10 \mathrm{keV} \mathrm{lu}$ minosity of MKN 231. This luminosity, which ranges between $4.5 \times 10^{43} \mathrm{erg} \mathrm{s}^{-1}$ and $2 \times 10^{44} \mathrm{erg} \mathrm{s}^{-1}$, is at least an order of magnitude higher than what has been previously measured.

We found that the observed flat $2-10 \mathrm{keV} \mathrm{X}$-ray continuum of MKN 231 can be explained assuming that below $10 \mathrm{keV}$ we are seeing only reprocessed radiation through reflection and/or scattering.

The starburst activity significantly contributes to the soft $(E<2 \mathrm{keV}) \mathrm{X}$-ray emission $\left(L_{\mathrm{Th}(0.5-2 \mathrm{keV})} \sim 3 \times 10^{41} \mathrm{erg} \mathrm{s}^{-1}\right)$, although a relevant contribution due to HMXB is also measured in the $2-10 \mathrm{keV}$ energy range $\left(L_{\mathrm{HMXB}(2-10 \mathrm{keV})}=\right.$ $\left.0.7-1.6 \times 10^{42} \mathrm{erg} \mathrm{s}^{-1}\right)$.

Note added in proof. After this paper was submitted, a detailed analysis of the iron $\mathrm{Fe} \mathrm{K} \alpha$ line was also posted on http://arXiv.org/abs/astro-ph/0308030 by Turner T. J. and Kraemer S. B. Their paper was then published in ApJ, 598, 916 (2003).

Acknowledgements. Based on observations obtained with XMM-Newton, an ESA science mission with instruments and contributions directly founded by ESA member states and USA (NASA). This research has made use of SAXDAS linearized and cleaned event files produced at the BeppoSAX Science Data Center, and the NASA/IPAC extragalactic database (NED). We thank all the members working at the above mentioned projects. We thank R. Saxton for his suggestions during the XMM-Newton data processing, and we thank L. Ballo, A. Caccianiga and the anonymous referees for useful comments. This work has received financial support from ASI (I/R/037/01) and from ASI (I/R/062/02) under the project "Cosmologia Osservativa con XMM-Newton".

\section{References}

Boella, G., Butler, R. C., Perola, G. C., et al. 1997, A\&AS, 122, 299

Braito, V., Franceschini, A., Della Ceca, R., et al. 2002, New Visions of the X-ray Universe in the XMM-Newton and Chandra Era, ESA-SP, ed. F. Jansen [arXiv: astro-ph/0202352]

Brandt, W. N., \& Gallagher, S. C. 2000, NewAR, 44, 461

Chiappetti, L., \& Dal Fiume, D. 1997, in Proc. of the 5th International Workshop on Data Analysis in Astronmy, ed. V. Di Gesù, M. J. B. Duff, A. Heck, et al., 101 
Dickey, J. M., \& Lockman, F. J. 1990, ARA\&A, 28, 215

Ehle, M., Breitfellner, M., Dahlem, M., et al. 2001, XMM-Newton Users' Handbook

Farrah, D., Afonso, J., Efstathiou, A., et al. 2003, MNRAS, 343, 585

Fiore, F., Guainazzi, M., \& Grandi, P. 1999, Cookbook for BeppoSAX NFI spectral analysis

Franceschini, A., Braito, V., Persic, M., et al. 2003, MNRAS, 343, 1181

Gallagher, S. C., Brandt, W. N., Chartas, G., \& Garmire, G. P. 2002a, ApJ, 567, 37

Gallagher, S. C., Brandt, W. N., Chartas, G., et al. 2002b, ApJ, 569, 655

George, I. M., Turner, T. J., Yaqoob, T., et al. 2000, ApJ, 531, 52

Gilfanov, M., Grimm, H. J., \& Sunyaev, R. 2004, MNRAS, 347, 57

Goldader, J. D., \& Joseph, R. D. 1995, ApJ, 444, 97

Green, P. J., Aldcroft, T. L., Mathur, S., Wilkes, B. J., \& Elvis, M. 2001, ApJ, 558, 109

Grimm, H.-J., Gilfanov, M., \& Sunyaev, R. 2003, MNRAS, 339, 793

Iwasawa, K. 1999, MNRAS, 302, 96

Krabbe, A., Colina, L., Thatte, N., \& Kroker, H. 1997, ApJ, 476, 98

Leahy, D. A., \& Creighton, J. 1993, MNRAS, 263, 314

Levenson, N. A., Weaver, K. A., \& Heckman, T. M. 2001, ApJ, 550, 230

Lonsdale, C. J., Lonsdale, C. J., Smith, H. E., \& Diamond, P. J. 2003 , ApJ, 592, 804

Magdziarz, P., \& Zdziarski, A. A. 1995, MNRAS, 273, 837

Maloney, P. R., \& Reynolds, C. S. 2000, ApJ, 545, L23
Mathur, S. 2000, MNRAS, 314, 17

Matt, G., Perola, G. C., \& Piro, L. 1991, A\&A, 247, 25

Matt, G., Fabian, A. C., \& Ross, R. R. 1993, MNRAS, 262, 179

Matt, G., Brandt, W. N., \& Fabian, A. C. 1996, MNRAS, 280, 823

Mewe, R., Gronenschild, E. H. B. M., \& van den Oord, G. H. J. 1985, A\&AS, 62, 197

Murray, N., Chiang, J., Grossman, S. A., \& Voit, G. M. 1995, ApJ, 451, 498

Persic, M., \& Rephaeli, Y. 2002, A\&A, 382, 843

Ptak, A., Heckman, T., Levenson, N. A., Weaver, K., \& Strickland, D. 2003, ApJ, 592, 782

Ranalli, P., Comastri, A., \& Setti, G. 2003, A\&A, 399, 39

Reeves, J. N., \& Turner, M. J. L. 2000, MNRAS, 316, 234

Risaliti, G., Gilli, R., Maiolino, R., \& Salvati, M. 2000, A\&A, 357, 13

Ross, R. R., \& Fabian, A. C. 1993, MNRAS, 261, 74

Ross, R. R., Fabian, A. C., \& Young, A. J. 1999, MNRAS, 306, 461

Sanders, D. B., \& Mirabel, I. F. 1996, ARA\&A, 34, 749

Smith, P. S., Schmidt, G. D., Allen, R. G., \& Angel, J. R. P. 1995, ApJ, 444, 146

Strüder, L., Briel, U., Dannerl, K., et al. 2001, A\&A, 365, L18

Taylor, G. B., Silver, C. S., Ulvestad, J. S., \& Carilli, C. L. 1999, ApJ, 519,185

Turner, M. J. L., Abbey, A., Arnaud, M., et al. 2001, A\&A, 365, L27

Turner, T. J. 1999, ApJ, 511, 142

Turner, T. J., \& Kraemer, S. B. 2003, ApJ, 598, 916

Yaqoob, T. 1997, ApJ, 479, 184

Zycki, P. T., \& Czerny, B. 1994, MNRAS, 266, 653 